\title{
Noise-Induced Linearisation
}

\author{
M I Dykman \\ Department of Physics, Stanford University, \\ Stanford, California 94305, USA. \\ D G Luchinsky ${ }^{[1]}$ \\ School of Physics and Materials, Lancaster University, \\ Lancaster, LA1 4YB, UK. \\ R Mannella \\ Dipartimento di Fisica, Università di Pisa, \\ Piazza Torricelli 2, 56100 Pisa, Italy. \\ P V E McClintock, H E Short, N D Stein and N G Stocks ${ }^{[2]}$ \\ School of Physics and Materials, Lancaster University, \\ Lancaster, LA1 4YB, UK.
}

\begin{abstract}
$\underline{\text { Abstract }}$
It is found that the response of a nonlinear dynamical system can be linearised, and its frequency dispersion diminished, by the addition of external noise of sufficient intensity. Taking as an example an overdamped bistable system driven by a low-frequency periodic field, this noise-induced linearisation is investigated through analogue electronic experiments. The wider implications are considered.
\end{abstract}

\footnotetext{
${ }^{[1]}$ Permanent address: All Russian Institute for Metrological Service, Andreevskaya nab 2, 117965 Moscow, Russia.

${ }^{[2]}$ Present address: Department of Engineering, University of Warwick, Coventry, CV4 7AL, UK.
} 


\section{INPUT}
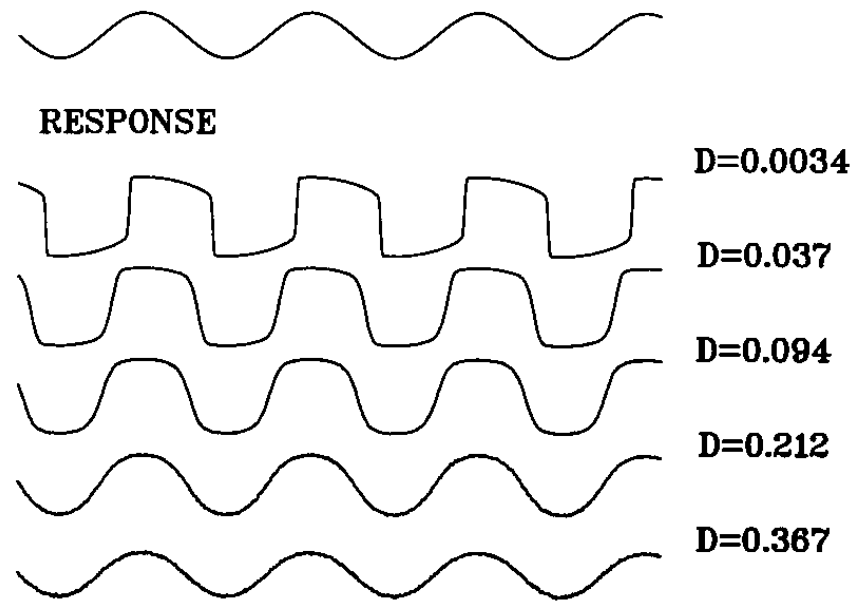

Figure 1: Noise-induced linearisation for a sine-wave passing through an electronic model of the overdamped double-well system given by Eqs.(4)-(6). The periodic force at the input is shown in the upper trace. The ensemble-averaged response $\langle q(t)\rangle$, measured at the output, is shown for different noise intensities $D$ in the lower traces. The amplitudes of the latter have been normalised so as to be comparable with the amplitude of the force, for easier comparison of their relative shapes.

When a signal passes through a nonlinear dynamical system, it emerges distorted. The severity of the effect depends on the frequency dispersion of the response and its nonlinearity, and on both the shape and the amplitude of the signal. We have observed, however, that this distortion can often be removed by the addition at the input of external white ${ }^{[3]}$ noise of sufficient intensity. The scenario has been found experimentally to hold for many nonlinear systems, including monostable as well as bistable, underdamped as well as overdamped, chaotic as well as regular, and for signals of various shapes. The process might be described by the term noise-induced linearisation. The linearised output $q(t)$ resulting from this procedure is inevitably noisy and so, to focus attention on what happens to the signal itself, in what follows we will discuss how the ensemble average $\langle q(t)\rangle$ of the output varies with relevant parameters, for example with the noise intensity at the input.

These ideas are illustrated by the experimental data shown in Figures 1 and 2, obtained from an analogue electronic circuit model of an overdamped bistable system driven by a periodic force (see below). The input to the system in each case is the waveform shown at the top, whose period is large compared to the relaxation time of the system to its stable state(s). For small (but not very small: see below) noise intensity $D$, the output response of the system is grossly distorted, as shown by the upper $\langle q(t)\rangle$ traces. As the noise intensity is increased, however, the distortion steadily diminishes in each case until, in the lowest trace, the output can be seen to be a faithful reproduction of the input waveform.

\footnotetext{
${ }^{[3]}$ For convenience, we will concentrate on the effects of white or quasi-white noise. Most of the discussion, however, is applicable qualitatively, and sometimes quantitatively, to the case of non-white noise, provided that its correlation time is shorter than the characteristic reciprocal frequencies of interest.
} 


\section{INPUT}

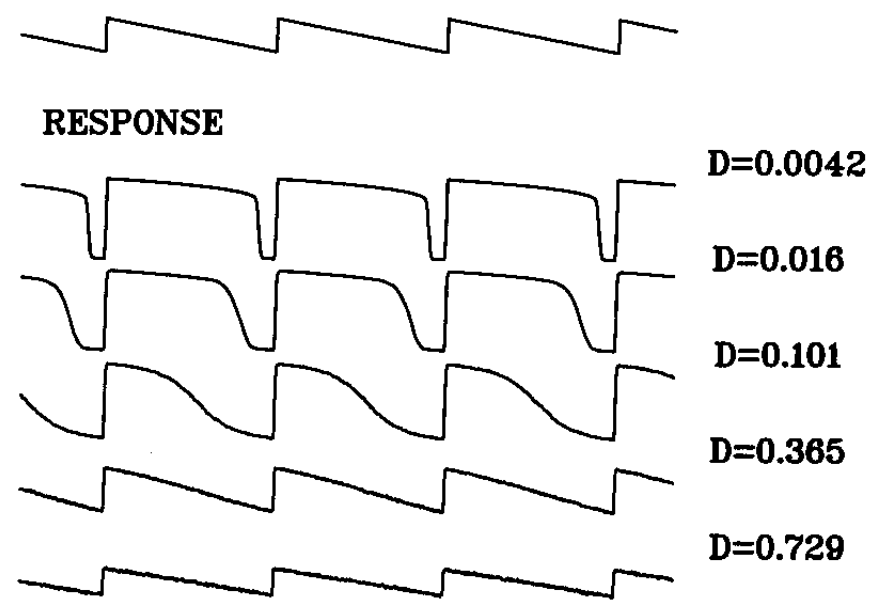

Figure 2: Noise-induced linearisation for a sawtooth wave passing through an electronic model of the overdamped bistable system given by Eqs.(4) - (6). The periodic force at the input is shown in the upper trace. The ensemble-averaged response $\langle q(t)\rangle$, measured at the output, is shown for different noise intensities $D$ in the lower traces. The amplitudes of the latter have been normalised so as to be comparable with the amplitude of the force, for easier comparison of their relative shapes.

The basic idea of linearisation by added noise will, of course, already be familiar through specific observations and applications in science and engineering, e.g. the linearisation of periodic signals in neurophysiological experiments [1], or the linearisation of the response of ring-laser gyroscopes at low angular velocities [2]. Our present results suggest, however, that noise-induced linearisation may exist as a more general phenomenon than has been appreciated, thus further illustrating the idea [3] that the role of noise in a dynamical system may in a certain sense be creative. Other examples that support this perception include: stochastic resonance [4] in which a weak periodic signal in a nonlinear system can be enhanced by noise; noise-induced transitions [5] where the number of extrema in a probability density varies with noise intensity; the creation of spatial structures in liquid crystals [6]; the stabilisation of spatial structures in convecting fluids [7]; arguably, the maintenance of consciousness [8]; and the stochastic ratchets that provide a possible basis for molecular locomotion [9] within biological cells.

We note that we use the word linearisation in two rather different senses, and that these are exemplified by the results of Figures 1 and 2. The fact that a sinusoidal input can pass through the system without significant change of shape, as occurs for strong noise in the lowest trace in Figure 1, implies linearity in the direct sense, i.e., a proportionality between the amplitudes of output and input; this need not necessarily, however, imply that the constant of proportionality must be frequency-independent. On the other hand, the results of Figure 2, for a sawtooth waveform containing not only the fundamental frequency but also its higher harmonics, imply the occurrence of linearisation in the "HiFi" sense that the system becomes non-dispersive within a certain frequency range when the noise intensity is large enough. In many cases of physical interest nonlinearity of the response arises because the frequency of the driving force (or its overtones) is close to 
some eigenfrequency of the system (or its overtones), or is related in a certain way to the relaxation time of the system. It is to be expected for such systems that linearisation of the response in the sense of proportionality of output and input will not necessarily imply suppression of the frequency dispersion. [4]

The physical origin of both forms of signal restoration can readily be understood, at least qualitatively, in the following terms. Where the amplitude response of a system to a periodic force is nonlinear, this arises because the amplitude of the vibrations induced by the force is comparable with, or larger than, some characteristic nonlinear length scale of the system. The scale in question (which may depend on frequency: see below) is determined by the structure of the region of phase space being visited by the system and by corresponding features in the dynamics. The effect of noise is to smear the system over a larger region of phase space, so that a variety of different scales and frequencies then become involved in the motion, even in the absence of periodic driving, and the effective characteristic scale will usually increase as a result. For sufficiently large noise intensities, therefore, the amplitude of the force-induced vibrations will become small compared to the scale (eg small compared to the average size of the noise-induced fluctuations), so that the nonlinearity of the response is correspondingly reduced. Because the system is then spending an increasing proportion of its time far away from its attractor(s), at coordinate values where the time that characterizes the motion will in general be quite different and sometimes shorter (or even much shorter: see below), than for small noise intensities, there will be one or more ranges of frequency for which dispersion is likely to decrease [10]. Although the linearisation and the suppression of the dispersion arise, ultimately, through the same physical processes - the effect of noise in smearing the system over a larger region of its phase space - there is no reason to expect that they will become important at the same noise intensity.

The harmonic oscillator provides an obvious example: here, the response is always linear, the amplitude of the forced vibrations being proportional to the amplitude of the force; at the same time, dispersion can be very strong, particularly for an underdamped oscillator in the range of its eigenfrequency. For nonlinear systems, the narrow peaks in spectral densities of fluctuations are eventually [11] broadened by an increase in noise intensity; for underdamped systems, one of the basic broadening mechanisms comes about because the eigenfrequencies of vibration depend on amplitude, so that the broader the distribution over amplitude becomes due to the increasing fluctuations, the broader becomes the distribution over eigenfrequencies [12].

Bistable systems display, in addition, a different and quite universal type of strong frequency dispersion. It arises at low frequencies (or at frequencies close to that of the strong force, if it is the latter that has created the bistability, as eg in optical bistability), both in underdamped and overdamped systems. It is related to the fact that a lowfrequency force can modulate the distribution of a bistable system over the states. This modulation has been investigated in great detail recently in the context of stochastic resonance [4]. It is effective when the frequency is low enough for one fluctuational transition in each direction to occur between the states, with high probability, during one period of the force.

\footnotetext{
${ }^{[4]}$ Obviously, one could try to devise special circumstances which might violate this rule. One possible example would be a logarithmic amplifier. However, even though this would be dispersion-free but highly nonlinear for low noise intensities, it seems likely nonetheless that the response would be eventually linearised by noise, just like the other cases that we consider.
} 
Quite generally, the ensemble-averaged term in the coordinate $\langle\delta q(t)\rangle$ of a stationary system that arises when the system is driven by a periodic force $A \cos \Omega t$ can be written in the form

$$
\langle\delta q(t)\rangle=\sum_{n} a(n)[\cos n \Omega t+\phi(n)]
$$

If the amplitude of the force $A$ is small enough, the response is linear, so that only the $n=1$ term need be retained:

$$
\langle\delta q(t)\rangle=a \cos (\Omega t+\phi)
$$

where

$$
a \equiv a(1)=A|\chi(\Omega)|, \quad \phi \equiv \phi(1)=-\arctan [\operatorname{Im} \chi(\Omega) / \operatorname{Re} \chi(\Omega)]
$$

Here, $\chi(\Omega)$ is the susceptibility of the system. It completely describes the linear response to a sufficiently weak periodic force, and it is the frequency dependence of $\chi(\Omega)$ that corresponds to the frequency dispersion of the linear response of a system. For systems performing Brownian motion of a quasi-thermal type, the fluctuation dissipation theorem [13] can be used to express the susceptibility $\chi(\Omega)$ in terms of the spectral density of fluctuations in the absence of the periodic force.

Where the input amplitude $A$ is not very small, however, the amplitudes $a(n)$ of vibration at the overtones $(n>1)$ will no longer be small and the system will display nonlinearity.

In the present Letter we illustrate noise-induced linearization and reduction of frequency dispersion for a particular bistable system. We consider as an example the case of overdamped Brownian motion in a symmetrical double well potential described by the equation

$$
\begin{gathered}
\dot{q}+U^{\prime}(q)=A \cos \Omega t+f(t) \\
U(q)=-\frac{1}{2} q^{2}+\frac{1}{4} q^{4}
\end{gathered}
$$

Here, $A \cos \Omega t$ is the periodic input force and $f(t)$ is quasi-white zero-mean Gaussian noise of intensity $D$,

$$
\left\langle f(t) f\left(t^{\prime}\right)\right\rangle=2 D \delta\left(t-t^{\prime}\right)
$$

The system (4)-(6) has been studied intensively in the context of stochastic resonance [4]; its effect in distorting rectangular signals has recently been discussed in [14]; and its properties are to a large extent understood. In particular, for small noise intensities, $D \ll \Delta U_{n}$, where $\Delta U_{n}\left(=\frac{1}{4}\right.$ for the model of Eq (5)) is the depth of the $n$th potential well $(n=1,2)$, the susceptibility of the system is given by the sum of the contributions from the motion about the minima of the wells that arises in response to the periodic force, and the contribution from the modulation of the populations of the stable states due to the force [15]

$$
\chi(\Omega)=\sum_{n} w_{n}\left(U_{n}^{\prime \prime}-i \Omega\right)^{-1}+\frac{w_{1} w_{2}}{D}\left(q_{1}-q_{2}\right)^{2} W(W-i \Omega)^{-1}
$$



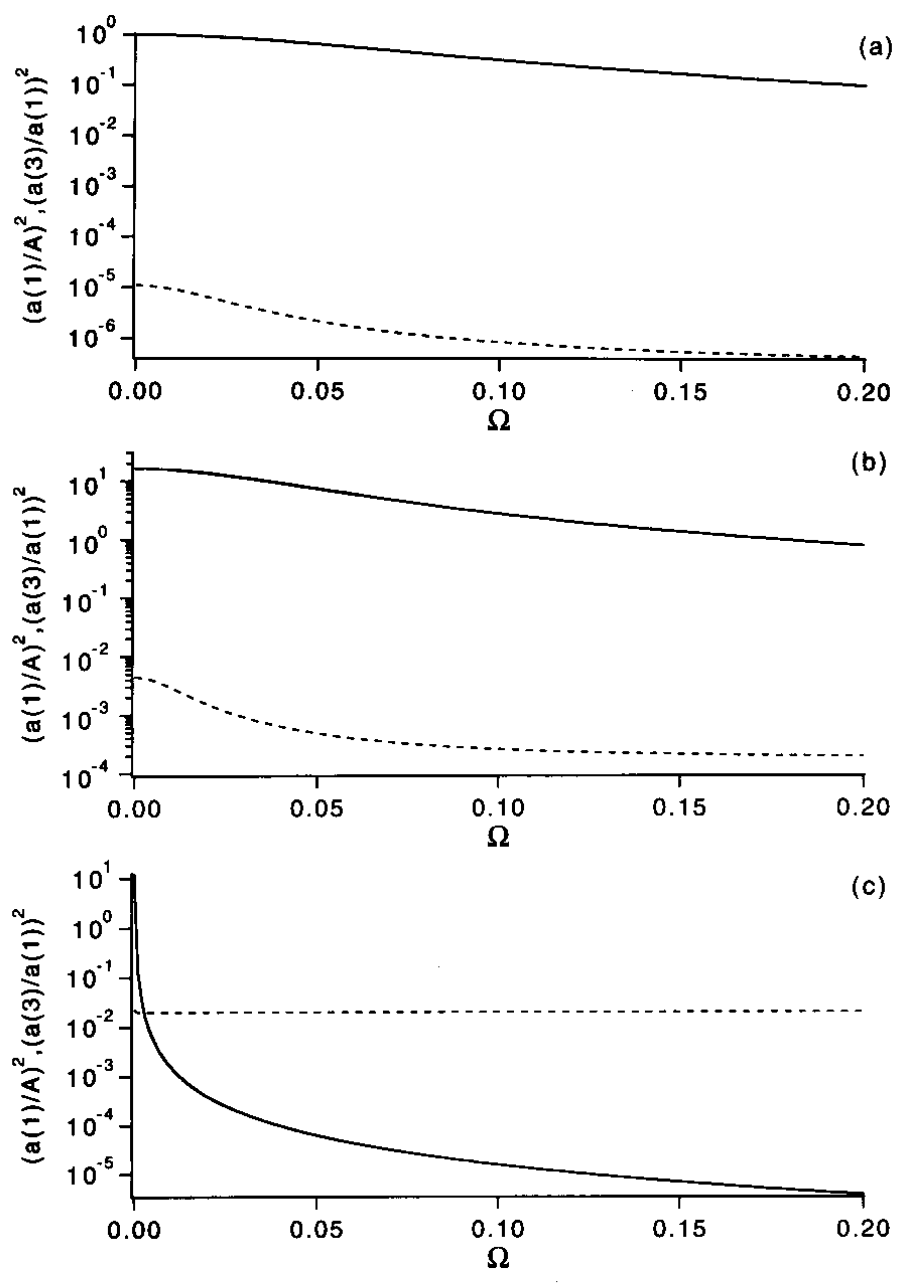

Figure 3: The normalised response of the system (7)-(9) to a sinusoidal driving force, as a function of its frequency $\Omega$, for three noise intensities: (a) $D=0.5$; (b) $D=0.3$; (c) $D=0.02$. The full curves represent the squared ratio of the response $a(1)$ at the driving frequency to the amplitude $A$ of the driving force $\left(=|\chi|^{2}\right)$. The dashed curves represent the squared ratio of the response $a(3)$ at the third harmonic of the driving frequency to the response $a(1)$ at the driving frequency.

where $w_{1,2}$ are the stationary populations of the wells $\left(w_{1}=w_{2}=\frac{1}{2}\right.$ for a symmetric potential), the $U_{n}^{\prime \prime}$ are the curvatures of the bottoms of the wells, and $W=W_{12}+W_{21}$ is the sum of the probabilities $W_{n m}$ of the interwell transitions $n \rightarrow m$.

The transition probabilities $W_{n m} \propto \exp \left(-\Delta U_{n} / D\right)$ increase exponentially fast with increasing noise intensity $D$ Therefore it is immediately evident from Eq. (7) that, for $\Omega \ll U_{n}^{\prime \prime}$, there will be strong dispersion for weak noise where $W \lesssim \Omega$ but negligible dispersion for stronger noise. Figure 3(a)-(c) shows the variation of the response with frequency, calculated [17] for three different noise intensities. The full curves correspond to the square of the response at $\Omega$ (see caption); they exhibit a strong dependence on frequency for weak noise intensity (c), but become much flatter as the noise intensity is increased to a larger value (a). We notice that the range of strong dispersion becomes exponentially narrow for very small noise intensities. 
Not only may bistable systems display strong frequency dispersion, but they are also known to display a giant nonlinearity $[15,16,17]$ where, for comparatively small input amplitudes $A$, the interwell transition probabilities are modulated strongly enough for the system to make on average almost exactly one pair of transitions, at nearly the same values of phase, on each cycle of the periodic force. Clearly, for very much lower noise intensities, such that the interwell transitions are suppressed, the response to weak periodic forcing about either one of the minima of the potential wells will be at least approximately linear. On the other hand, for high noise intensities many transitions will happen at random over the period of the force, and they will not be synchronized. Consequently, the parameter range where the system displays the giant nonlinearity in response to a small-amplitude periodic force is limited to low frequencies $\Omega$ and appropriately small noise intensities.

One may choose as a quantitative measure of the weakness (or otherwise) of the nonlinearity of the response of a system the ratio of the harmonics $a(n) / a(1)$ in (1). The dashed curves in Fig. 3 indicate the squared ratio of the third harmonic to that at the fundamental, $[a(3) / a(1)]^{2}$ (by symmetry arguments, $a(2)=0$ for the system (4) (6)). It is clear that these nonlinear contributions decrease rapidly with increasing noise intensity. Noise-induced linearisation (in both the amplitude and frequency senses) is therefore indeed to be anticipated for the system (4) - (6), thereby accounting for the phenomena observed in Figures 1 and 2 which were obtained from an analogue electronic circuit of conventional $[18,19]$ design built to model this system.

It is important to note, and it is clear from the above example, that the same physical processes which give rise to noise-induced linearisation can also, under special circumstances, give rise to the opposite effect of noise-induced delinearisation. The latter phenomenon is to be anticipated if the additional frequencies that become involved (as the result of an increase in noise intensity) resonate with the periodic force or with one of its harmonics, or if the noise modifies the characteristic reciprocal relaxation time of the system so that it corresponds to the frequency of the periodic force. In such cases, just as seen above for the system (4) - (6), it is to be expected that the promotion of nonlinearity by noise at intermediate intensities will be followed by the more general phenomenon of noise-induced linearisation at still higher noise intensities owing to the usual noise-induced broadening of the relevant spectral peak(s). We have also observed and investigated sequential noise-induced delinearization/linearisation phenomena in dynamical systems quite different from (4) - (6), for example, in an underdamped monostable oscillator [10].

We conclude that the noise-induced linearisation of the response of a nonlinear system to a sinusoidal force is a quite general phenomenon. Noise-induced suppression of the frequency dispersion for low frequencies also appears to be of wide occurrence: we suggest that it is to be anticipated for Brownian motion in all potentials that are harder (i.e. rising faster) than parabolic within the relevant energy range, provided that the fundamental frequency of the force and its relevant harmonics are very much smaller then the natural frequency of small oscillations in underdamped systems, or than the reciprocal relaxation time in the case of overdamped systems. A sufficient condition for removing low-frequency dispersion, in the case of simple, non-chaotic, attractors (and for non-potential motion, generally) appears to be that the time needed to come from infinity should be finite. The delinearisation phenomenon is likely to arise in those cases where the frequency of the periodic force, or of one of its harmonics, can be tuned by the noise close to an eigenfrequency (or in certain cases a reciprocal characteristic relaxation time) of the 
system.

\section{ACKNOWLEDGEMENTS}

We gratefully acknowledge partial support of this work by the Science and Engineering Research Council (UK), by the Royal Society of London, by the European Community Directorate General XII and by the Nuffield Foundation (London).

\section{References}

[1] A. S. French, A. V. Holden and R. B. Stein, Kybernetik 11 (1972) 15; see also J. P. Segundo, J.-F. Vibert, K. Pakdaman, M. Stiber and O. Diez Martinez, "Noise in the neurosciences: a long history, a recent revival and some theory", in K. Pribram, ed. Proceedings of the Second Appalachian Conference, in press, and references therein.

[2] K. Vogel, H. Risken, W. Schleich, M. James, F. Moss, R. Mannella and P.V.E. McClintock, J. Appl. Phys. 62 (1987) 721.

[3] M.M. Millonas, ed., Fluctuations and Order: the new Synthesis, Springer, Berlin, in press.

[4] See J. Stat. Phys. 70, nos. 1/2 (1993), special issue on stochastic resonance, ed. F. Moss, A. Bulsara and M.F. Shlesinger.

[5] W. Horsthemke and R. Lefever, Noise-Induced Transitions (Springer-Verlag, Berlin, 1984).

[6] S. Kai, "Electrohydrodynamic Instability of Nematic Liquid Crystals: Growth Process and Influence of Noise", In Noise in Nonlinear Dynamical Systems, edited by F. Moss and P.V.E. McClintock, volume 3, 22-76 (Cambridge University Press, Cambridge, 1989).

[7] R.J. Deissler, J. Stat. Phys. 54 (1989) 1459.

[8] A.J. Mandell and K.A. Selz, J. Stat. Phys. 70 (1993) 355.

[9] M.O. Magnasco, Phys. Rev. Lett. 72 (1994) 2656.

[10] M.I. Dykman, D.G. Luchinsky, R. Mannella, P.V.E. McClintock, H.E. Short, N.D. Stein and N.G. Stocks, "Noise-induced linearisation and delinearisation", in M.M. Millonas, ed., Fluctuations and Order: the New Synthesis, Springer, Berlin, in press.

[11] M.I. Dykman, R. Mannella, P.V.E. McClintock, S.M. Soskin and N.G. Stocks, Phys. Rev. A 42 (1990) 7041.

[12] M.I. Dykman and M.A. Krivoglaz, in Soviet Physics Reviews, ed by I.M. Khalatnikov (Harwood, New York 1984) vol.5, p. 265; M.I. Dykman and P.V.E. McClintock, Physica D 58, 10 (1992).

[13] L.D. Landau and E.M. Lifshitz, Statistical Physics, 3rd Edition (Pergamon, Oxford, 1980). 
[14] M. Morillo and J. Gòmez Ordòñez, "Amplification and distortion of a periodic rectangular driving signal by a noisy bistable system", to be published.

[15] M.I. Dykman, R. Mannella, P.V.E. McClintock, and N.G. Stocks, Phys. Rev. Lett. 65 (1990) 2606; M.I. Dykman, P.V.E. McClintock, R. Mannella, and N.G. Stocks, Sov. Phys. J.E.T.P. Lett. 52 (1990) 141.

[16] T. Zhou, F. Moss and P. Jung, Phys. Rev. A 42 (1990) 3161.

[17] M.I. Dykman, R. Mannella, P.V.E. McClintock, N.D. Stein and N.G. Stocks, Phys. Rev. E 47 (1993) 1629.

[18] L. Fronzoni, "Analogue Simulations of Stochastic Processes by Means of Minimum Component Electronic Devices." In Noise in Nonlinear Dynamical Systems, edited by F. Moss and P.V.E. McClintock, volume 3, 222-242 (Cambridge University Press, Cambridge, 1989).

[19] P.V.E. McClintock, and F. Moss, "Analogue Techniques for the Study of Problems in Stochastic Nonlinear Dynamics." In Noise in Nonlinear Dynamical Systems, edited by F. Moss and P.V.E. McClintock, volume 3, 243-274 (Cambridge University Press, Cambridge, 1989). 
FIGURE CAPTIONS 